\title{
Transformada wavelet y máquinas de soporte vectorial para la identificación de arritmias
}

\section{Wavelet transform and support vector machines for the arrhythmia identification}

\author{
Diego Alejandro Tovar Salazar*, Alejandro José Orozco Naranjo*, Pablo Andrés Muñoz Gutiérrez**, \\ Héctor Murillo Wills***, Javier Alejandro Granada****
}

Recibido: Febrero 13 de 2009

Aceptado: Septiembre 1 de 2009

Correspondencia: Programa de Ingeniería Electrònica, Universidad del Quindio, Avenida Bolívar calle 12 norte, Armenia Quindio, Email: gama@uniquindio.edu.co

\section{Resumen}

Se presentan varios conjuntos de extracción de características de latidos normales y latidos de cuatro tipos de arritmias cardíacas, usando la transformada wavelet. Un grupo de conjuntos elegidos aleatoriamente, fueron utilizados para determinar los parámetros óptimos de la función núcleo polinomial (C y d) y de la función núcleo de base radial (Cy gamma), que arrojaran los mejores resultados de clasificación para las máquinas de soporte vectorial. A partir de estos parámetros óptimos se realizaron pruebas de clasificación con todos los conjuntos de entrenamiento y se obtuvo un porcentaje de error de validación del 2.93\% para una máquina de soporte vectorial con función núcleo de base radial, $11.72 \%$ con un clasificador Bayesiano y $3.63 \%$ con un perceptrón multicapa. Con el fin de reducir el porcentaje de error de validación, se aplicó la técnica de análisis por componentes principales para la selección efectiva de características y para reducir la dimensionalidad de los vectores de características de cada conjunto de entrenamiento. Las máquinas de soporte vectorial evaluadas con estos nuevos conjuntos de entrenamiento, fueron más consistentes y presentaron siempre un porcentaje de error de validación menor, logrando un porcentaje de error de validación del 1.93\%, el clasificador Bayesiano mejoró sustancialmente su capacidad de clasificación en la mayoría de casos, mientras que el perceptrón multicapa no fue muy susceptible a la selección efectiva de características y en algunos casos mejoró y en otros no.

Palabras claves: Arritmias cardíacas, Electrocardiografía, Wavelet, Máquina de Soporte Vectorial, Perceptron Multicapa.

\begin{abstract}
In this paper we present several characteristics extraction schemes set of both normal beatings and those with four different types of cardiac arrhythmias through the wavelet transform. One of these group schemes, randomly chosen, was used to determine the optimal parameters of the Polynomial Kernel ( $C \& D)$, and the radial basis kernel (C \& gamma) from which we could obtain the best classification results for the Support Vector Machines. According to these parameters we applied different classification tests with all the training set, which led us to an error validation percentage of 2.93 with a support vector machine with a radial basis kernel, 11.72 with a Bayesian Classifier, and 3.63 with a multilayer perceptron. With the purpose of reducing the error validation percentage, we applied the principal components analysis technique for both the effective selection of characteristics and the reduction of the dimensionality of the characteristic vectors on each training set. As a result, we could observe that the support vector machines evaluated with these new training set were the most consistent, having a lower error validation percentage of 1.93; and the Bayesian classifier improved its classification ability, while the multilayer perceptron did not have an accurate response to the effective selection of characteristics.
\end{abstract}

Keys Words: Cardiac arrhythmias, Electrocardiography, Wavelet, Support Vector Machine, Multilayer Perceptron.

\footnotetext{
* Implementación de un laboratorio de electrofisiología para el estudio de enfermedades coronarias. Proyecto de investigación \#346 Estudiante de Ingeniería Electrónica de la Universidad del Quindío.

** Programa de Ingenería electrónica. Universidad del Quindìo.

***Programa de Medicina. Universidad del Quindìo.

**** Estudiante de Medicina de la Universidad del Quindío.
} 


\section{INTRODUCCIÓN}

os sistemas de reconocimiento están en carrera de alcanzar el punto en el cual tengan la capacidad de apoyar en gran medida el diagnóstico médico. El registro electrocardiográfico (ECG) es la representación gráfica del comportamiento ventricular y auricular del corazón, ha sido de gran importancia para el cuidado de la salud humana y en él se han centrado un sinnúmero de trabajos de investigación que buscan determinar que señales son normales o anormales [1] y [2].

El funcionamiento del músculo cardiaco se caracteriza por tener una enorme variabilidad. Cuando se observa una señal cardiaca se tienen componentes de interés como son: frecuencia, ritmo, ejes, magnitud; en el registro gráfico se puede observar el momento donde se produce la repolarización y despolarización del músculo. La mayoría de trabajos revisados utilizan la MIT BIH Arrhythmia Database, que pertenece al BIH Arrhythmia Laboratory, con el fin de realizar pruebas tanto de sistemas para el procesamiento de señales cardiacas como para validar sistemas de identificación de patrones anormales en estas [3], [4], [5] y [6]. El primer paso de este trabajo fue orientado a obtener y analizar las señales cardiacas de los registros de la base de datos de arritmias, a través del programa rdann.m disponible en Physionet.

La transformada wavelet es un gran aporte matemático para el análisis y procesamiento de señales y ha tenido una gran aplicación en el desarrollo de sistemas para el análisis, procesamiento y clasificación de señales biológicas [5], [7] y [8]. Para cualquier sistema de clasificación es de gran importancia obtener los puntos representativos de la señales a identificar; por tanto la transformada wavelet se usó como metodología para la extracción de las características. Gracias a la diversidad de wavelets madre y mediante la descomposición en diferentes niveles de detalles y aproximaciones se logró evaluar un grupo amplio de estructuras de características para la identificación de las arritmias analizadas.

Las máquinas de soporte vectorial surgen como algoritmos de aprendizaje alternativos para clasificadores polinomiales, de funciones de base radial o para el perceptrón multicapa; los sistemas de aprendizaje transforman el vector característico de entrada, a través de alguna transformación no lineal a priori, a un espacio de características de mayor dimensión, donde pueda ser tratado como un problema de clases linealmente separables [9].

Con el fin de optimizar el proceso de clasificación, se evaluaron metodologías que reduzcan el tamaño de los vectores de características; la técnica de análisis por componentes principales (PCA - Principal Components Analysis) realiza una transformación para eliminar la información redundante contenida en el vector de características, disminuyendo su tamaño de conjunto [6].

De acuerdo al estudio realizado por otros grupos de investigación en ésta área, han sido documentados errores de clasificación pequeños al emplear técnicas para selección efectiva de características, en el artículo [5] el error de clasificación oscila entre $0 \%$ y $2 \%$, en [4] se menciona un error del 0.05 aproximadamente. Los resultados de los artículos mencionados son válidos para una máquina de aprendizaje entrenada solo para la clasificación de dos clases; en este trabajo se contempla la clasificación de cinco clases, que consisten en cuatro arritmias cardiacas y la señal normal del latido cardiaco. En la siguiente parte de este trabajo se describe la metodología empleada y con detalle se analiza la selección de los latidos a estudiar, los Esquemas de extracción de características y los sistemas de aprendizaje, los resultados serán mostrados en la última parte; finalmente en la conclusiones se discute sobre estos resultados y se plantean ideas sobre los esquemas desarrollados.

\section{METOdOLOGÍA}

El Diagrama de Bloques de la Figura 1 describe la metodología utilizada para la identificación de arritmias cardiacas usando la transformada wavelet y máquinas de soporte vectorial (MSV).

\section{Bade de datos}

Los ejemplos de latidos para cada clase se extrajeron de la base de datos de arritmias cardiacas de la MIT BIH Arrhythmia Database; los registros incluyen señales normales y diferentes arritmias cardiacas. Se utilizó la derivación V1 por ser de las de mayor uso en el diagnostico médico. El origen de la señal tomada se encuentra en las células del músculo cardiaco, las cuales pueden ser excitadas eléctricamente [7]. El término arritmia es muy general y se refiere específicamente a todos los otros ritmos diferentes al ritmo normal del corazón [11]. Las arritmias se producen tanto en los ventrículos como en las aurículas, cada una de ellas presenta formas características.

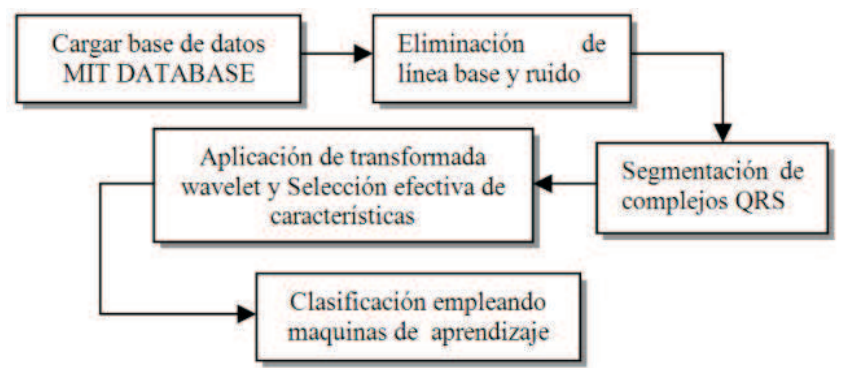

Figura 1. Diagrama de bloques de la metodología empleada 
La investigación se enfocó en el análisis morfológico de 5 latidos cardiacos: Normal (N), latido LBBB (Left Bundle Branch Block- Bloqueo de Rama Izquierda), latido RBBB (Right Bundle Branch Block- Bloqueo de Rama Derecha), latido PVC (Premature Ventricular Contraction - Contracción Prematura Ventricular), latido APC (Atrial Premature Contraction-Contracción Prematura Auricular).

\section{Filtrado de la Señal}

Las señales electrocardiográficas presentan ruidos característicos como son el ruido térmico, de red eléctrica y de línea base; los cuales deben ser filtrados para garantizarle al médico especialista y al sistema de clasificación registros con la menor presencia de ruido. Aunque los registros ECG utilizados presentan una baja presencia ruido, se diseñaron filtros para suavizar cualquier componente distorsionador en las señales.

Se deben emplear técnicas de filtrado con la capacidad de adaptarse dependiendo del tipo de señal a tratar. En general los métodos propuestos para filtrar ruido de naturaleza gausiana como es el ruido térmico, utilizan transformaciones de la señal (transformada wavelet) y operan sobre los coeficientes producidos. Esta técnica consiste en la determinación de un nivel de umbralización para disminuir los componentes que producen perturbación y finalmente calcular la transformada inversa para recuperar la señal [3].

En ésta metodología de filtrado se deben especificar parámetros como: El tipo de umbralización, la wavelet madre, la estimación de ruido y el escalamiento al ruido estimado. Los parámetros empleados respectivament fueron: Soft, Daubechies 4, Rigsure y SLN respectivamente, los cuales fueron establecidos tras un análisis previo y otros resultados de investigación [1], [7] y [11]. En lo relacionado a la eliminación de ruido de línea base se emplea un filtro de media móvil cuya ventana depende de la cantidad total de muestras en el registro analizado [12].

\section{Segmentación}

La segmentación consiste en tomar muestras presentes en el registro ECG donde estén presentes los complejos QRS, para luego analizarlos utilizando la transformada wavelet. Para cumplir con el objetivo se emplea el software libre disponible en la página web de MIT BIH Arrhythmia Database, como es el rdann.m, el cual se encarga de cargar los archivos donde están almacenados los registros ECG. Al cargar dichos archivos se encuentran los valores eléctricos de dos derivaciones la MLII y la V1, pero se seleccionó para el análisis sólo la derivación V1. Éstos también cuentan con otras dos variables entregadas en vectores, el primero contiene el tiempo transcurrido y el otro las anotaciones; en estasanotaciones se demarca numéricamente el tipo de cada latido, ver Figura 2.

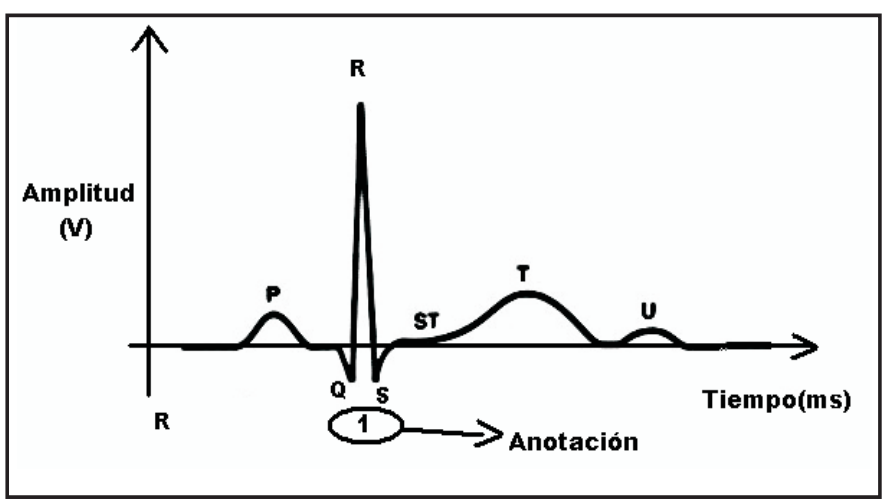

Figura 2. Representación de un complejo QRS y la ubicación de las anotaciones.

Imagen extraída y adaptada de la original en (Balomenos $\mathrm{T}$. et al, 2002).

Se hizo un análisis previo de la cantidad de latidos cardiacos pertenecientes a las arritmias disponibles, y se seleccionaron las cinco que presentaran un mínimo de 1180 ejemplos, todas las señales forman un conjunto para extracción de características de 5900 latidos, en la Figura 3 se muestran ejemplos de cada arritmia.

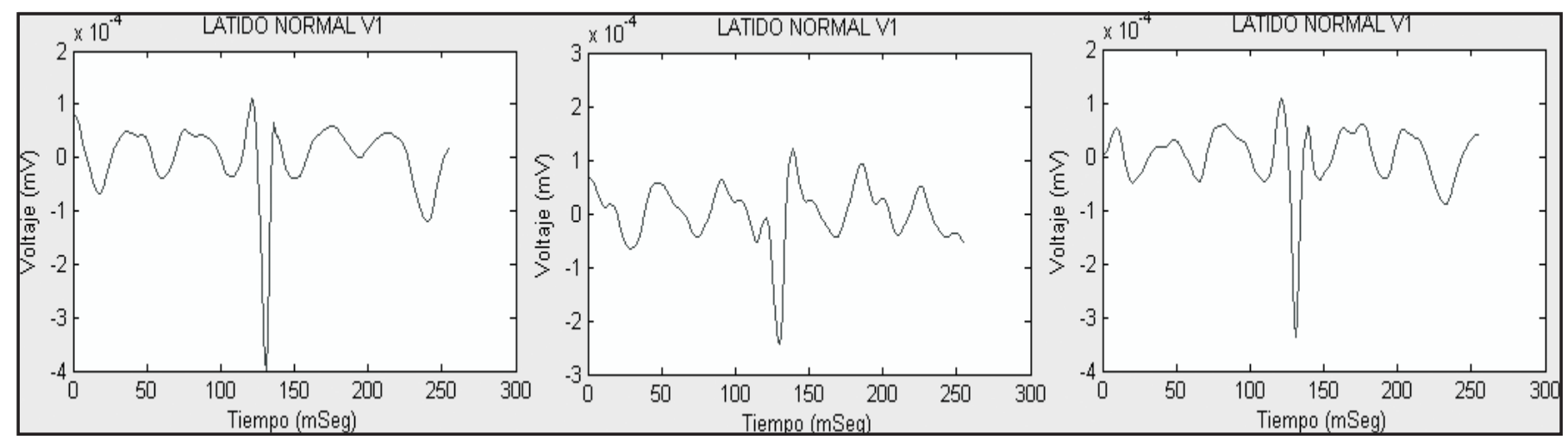

Figura 3. Ejemplos de latidos estudiados 

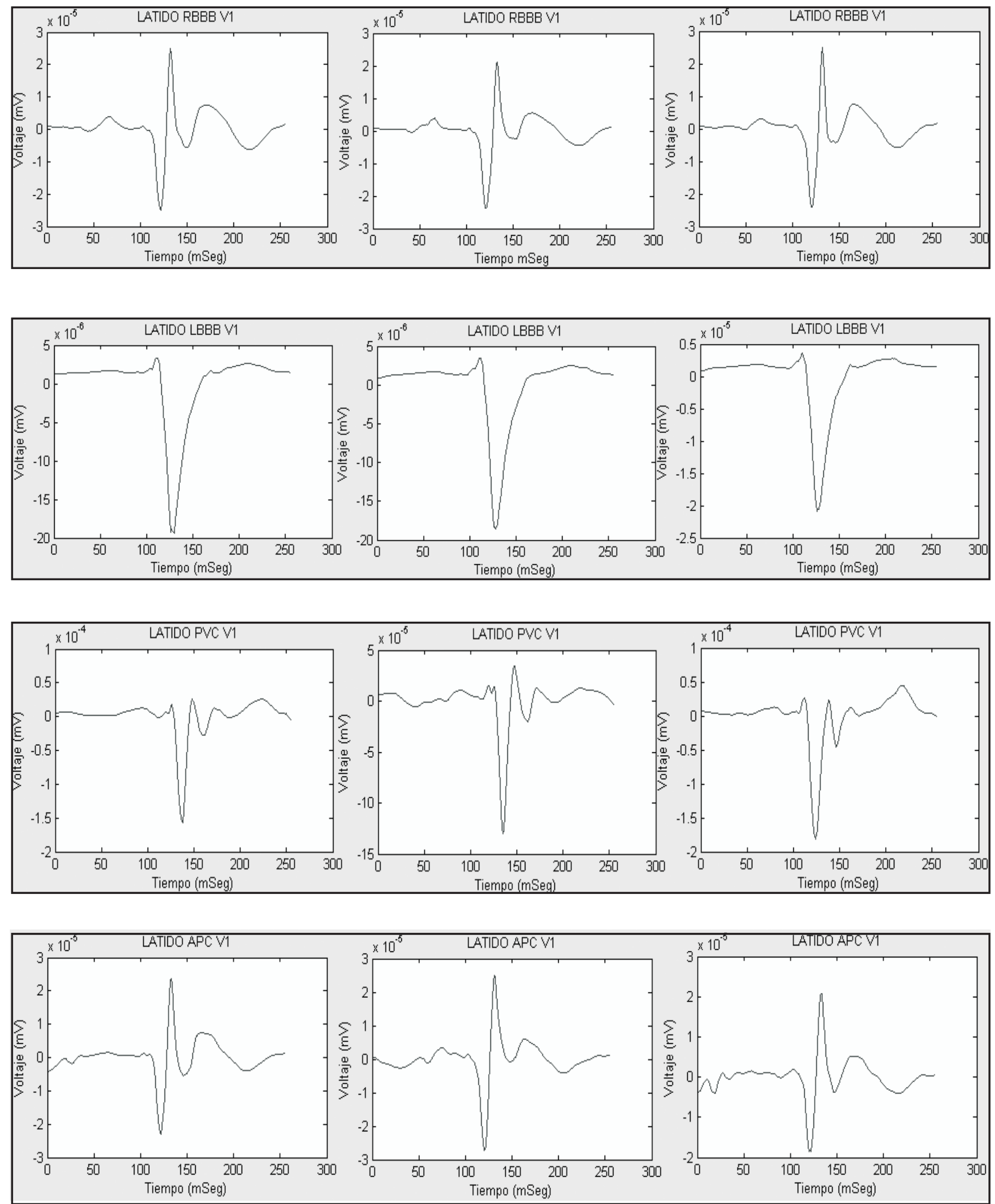

Figura 3. Ejemplos de latidos estudiados 
El proceso de segmentación consiste en buscar una anotación en particular, en el momento que dicha anotación es encontrada, se procede a contar cuanto tiempo hay desde el pico correspondiente a dicha anotación, hasta el siguiente pico indicador de una nueva anotación; de igual manera se procede para determinar el tiempo de separación entre la anotación encontrada y la anotación inmediatamente anterior. Después de calcular estos tiempos, se multiplican por 0.5 y con ese par de límites, uno a la derecha y otro a la izquierda, se garantiza que ha quedado enventanado el complejo QRS al cual corresponde la anotación buscada.

\section{Extracción de Características}

La transformada wavelet fue la herramienta utilizada para obtener las características y construir los esquemas de entrenamiento utilizados para evaluar las máquinas de aprendizaje. El objetivo de la transformada wavelet es descomponer la señal tratada en un conjunto de señales restringidas para ciertas bandas de frecuencia, las cuales forman una base del espacio de funciones, con ciertas propiedades como ortogonalidad, tamaño, suavidad, etc. La descomposición se realiza a partir de funciones más complejas, en las cuales no se varía la frecuencia, sino su posición y su escala temporal.

Cuando se hace referencia al término wavelet madre, se está indicando que las funciones usadas, con diferente zona de acción, derivan de una función principal, la wavelet madre es un prototipo a partir del cual se generan el resto de funciones de enventanado [7].

Definición Transformada wavelet discreta: Sea la señal a analizar $f[n]$ una función discreta. En este caso la transformada Wavelet de esta señal viene dada por:

$$
C[j, k]=\sum_{n \in Z} f[n] \psi_{j, k}[n]
$$

Donde $\mathrm{Y}_{\mathrm{j}, \mathrm{ky} \text { es una Wavelet discreta definida como: }}$

$$
\psi_{j k}[k]=2^{-\frac{1}{2}} \psi\left[2^{-j} n-k\right]
$$

Los parámetros $a, b j$ están definidos según la escala diádica, de manera que $a=2, b=2 b k$, con la diferencia respecto a la SWT que la señal bajo estudio es discreta. La transformada inversa se define de forma similar como:

$$
f[n] \sum_{j \in z} \sum_{k \in Z} c[j, k] \psi_{j k}[n]
$$

\section{Análisis de Multi-resolución:}

Se desarrolló para descomponer señales de tipo discreto. Su idea consiste en tener una representación tiempo-escala de una señal discreta. La señal se pasa a través de filtros paso alto para analizar las componentes de alta frecuencia, y se pasa a través de filtros paso bajo para analizar las componentes de baja frecuencia, ver Figura 4. Estas operaciones cambian la resolución de la señal, y la escala se cambia mediante operaciones de interpolación y submuestreo. Los términos producidos tras la transformación son conocidos como coeficientes de aproximación y coeficientes de detalle.

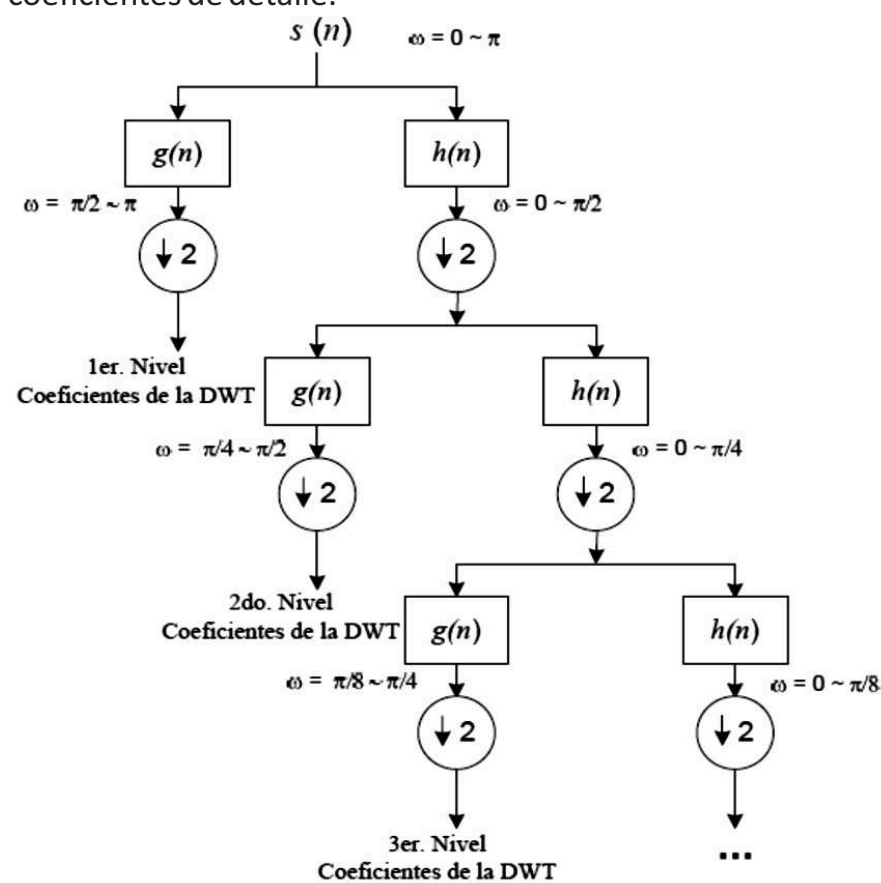

Figura 4. Algoritmo piramidal. La parte superior es la de análisis con $\mathrm{H}$ siendo el filtro paso bajo y $\mathrm{G}$ el filtro paso alto, mientras que la inferior es la síntesis. Fuente: [3]

Para la selección de la wavelet madre se tuvieron criterios como: Forma de onda, que debe tener una similitud con la señal ECG, la cantidad de singularidades que se presenten en la señal. En el caso que se tengan pocas singularidades, la mejor opción es una wavelet madre con altos momentos de desvanecimientos para asegurar un análisis más exacto. En cambio si la cantidad de singularidades se incrementa por unidad de tiempo se debe hacer uso de una wavelet madre con menor número de desvanecimientos, para evitar un mayor coste computacional [8].

La transformada wavelet trabaja con niveles de descomposición, para la investigación se usaron 6 niveles de acuerdo a las wavelet madre usadas y a la cantidad demuestras por cada señal. En la Tabla 1 se ilustran las wavelet madres utilizadas. 
Tabla 1. Wavelet Madre (Disponible: http://wavelets.pybytes.com)

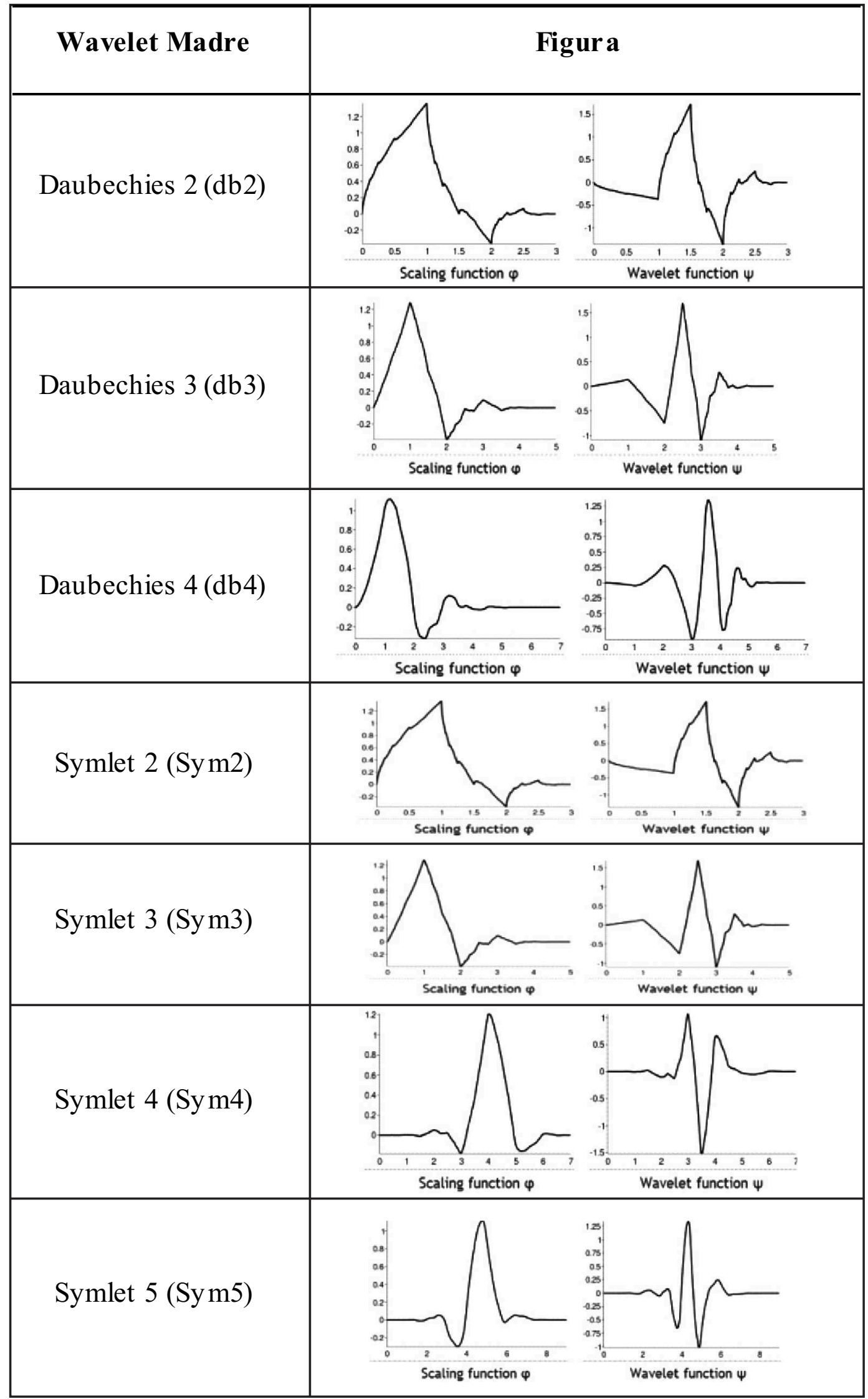


Después de aplicar la transformada wavelet a las señales electrocardiográficas se generaron los conjuntos de entrenamiento seleccionando características de los coeficientes de aproximaciones y de detalles de todos los niveles de descomposición.

Se construyeron 30 Esquemas de entrenamiento a los que se aplicaron las 7 wavelets madres seleccionadas, obteniendo 210 conjuntos de entrenamiento.

La Tabla 2 contiene 14 conjuntos de entrenamiento con la cantidad de niveles de descomposición, tipo de coeficientes utilizados, wavelet madre aplicada y la quinta columna muestra el número de características para cada ejemplo a clasificar. Se analizaron los puntos más representativos en la descomposición wavelet de cada tipo de arritmia cardiaca y en los latidos normales, buscando que indicaran diferenciabilidad entre las clases.

\section{Selección Efectiva de Características}

El Análisis por Componentes Principales (PCA), es una técnica para la reducción dimensional bajo el criterio del error cuadrático y es de gran uso para la selección efectiva de características. Se busca reducir la dimensión de la información, encontrando combinaciones lineales ortogonales de los datos originales con las varianzas más grandes.

Esto es, dado el coeficiente $s 1=x$ w1, donde el vector $p$ dimensional es,

$$
w_{1}=\operatorname{argmax}_{\|w=1\|} \operatorname{var}\left\{x^{T} w\right\}
$$

La técnica de PCA se aplicó para reducir el número de características de las matrices de entrenamiento (donde están contenidas las características de los ejemplos), llevando los conjuntos de entrenamiento a dimensiones $15 \times 5900$, garantizando que en este nuevo conjunto se tuvieran varianzas acumuladas entre el $85 \%$ y el $99 \%$, donde se puede afirmar que está concentrada la información más significativa de cada ejemplo. Con esta técnica no sólo se disminuye la dimensionalidad del vector de características (quinta columna de la tabla 2), también se reduce la redundancia en la información, mejorándose por lo tanto los resultados entregados por las máquinas de aprendizaje. La sexta columna de la Tabla 2, muestra las varianzas acumuladas logradas con 15 componentes principales para algunos conjuntos de entrenamiento.

Tabla 2. Cantidad de datos y varianzas acumuladas por conjunto de estudio

\begin{tabular}{|c|c|c|c|c|c|}
\hline Viveles & Coeficientes & Caracterís ticas & $\begin{array}{l}\text { Wavelet } \\
\text { madre }\end{array}$ & $\begin{array}{l}\text { Dimensión del } \\
\text { vector de } \\
\text { características }\end{array}$ & $\begin{array}{l}\text { Varianza } \\
\text { acumulada }\end{array}$ \\
\hline 1 al 6 & $\begin{array}{l}\text { Aproximaciones y } \\
\text { detalles }\end{array}$ & $\begin{array}{c}\text { 6 Mínimos y } \\
\text { Aproximaciones de cada } \\
\text { nivel }\end{array}$ & $\mathrm{db} 2$ & 72 & 96.07 \\
\hline $1 \mathrm{al} 6$ & Aproximaciones & 4 mínimos de cada nivel & $\mathrm{db} 2$ & 24 & 99.32 \\
\hline 1 al 6 & Aproximaciones & 6 Máximos de cada nivel & $\mathrm{db} 3$ & 36 & 99.08 \\
\hline 1al 6 & Aproximaciones & 6 mínimos de cada nivel & $\mathrm{db3}$ & 36 & 99.04 \\
\hline $1 \mathrm{al} 6$ & Detalles & 5 Mínimos de cada nivel & sym2 & 30 & 98.06 \\
\hline 1 al 6 & Aproximaciones & 5 Máximos de cada nivel & sym2 & 30 & 97.96 \\
\hline 1 & Aproximaciones & Todos los coeficientes & sym3 & 130 & 85.08 \\
\hline 1 al 6 & Detalles & Máximos de cada nivel & sym3 & 36 & \\
\hline 1 al 6 & $\begin{array}{c}\text { Aproximaciones y } \\
\text { detalles }\end{array}$ & 6 mínimos de cada nivel & sym4 & 72 & 96.07 \\
\hline $1 \mathrm{al} 6$ & Aproximaciones & 4 mínimos por cada nivel & sym4 & 24 & 99.31 \\
\hline 1 al 6 & Detalles & $\begin{array}{c}3 \text { Mínimos y } 3 \text { Máximos } \\
\text { de cada nivel }\end{array}$ & sym5 & 36 & 96.04 \\
\hline 1 al 6 & Detalles & 4 mínimos de cada nivel & sym5 & 24 & 98.65 \\
\hline
\end{tabular}




\section{Clasificación usando máquinas de soporte vectorial}

Para la clasificación se entrenaron y validaron tres máquinas de aprendizaje (MA) diferentes: El perceptrón Multicapa (MLP), las máquinas de soporte vectorial (SVM) y un clasificador Bayesiano. La comparación de los resultados entregados por las máquinas de aprendizaje mencionadas se realizó con el fin de analizar el comportamiento de los diferentes esquemas de extracción de características sobre los sistemas de aprendizaje, pero con especial interés en los resultados logrados con las máquinas de soporte vectorial, de las que se tienen buenas expectativas. La idea principal de las máquinas de soporte vectorial, es realizar una transformación no lineal () a priori del espacio de entrada a un espacio de características de dimensión mayor, donde posiblemente los datos pueden ser tratados de forma lineal, la Figura 5 ilustra un poco este proceso [9].

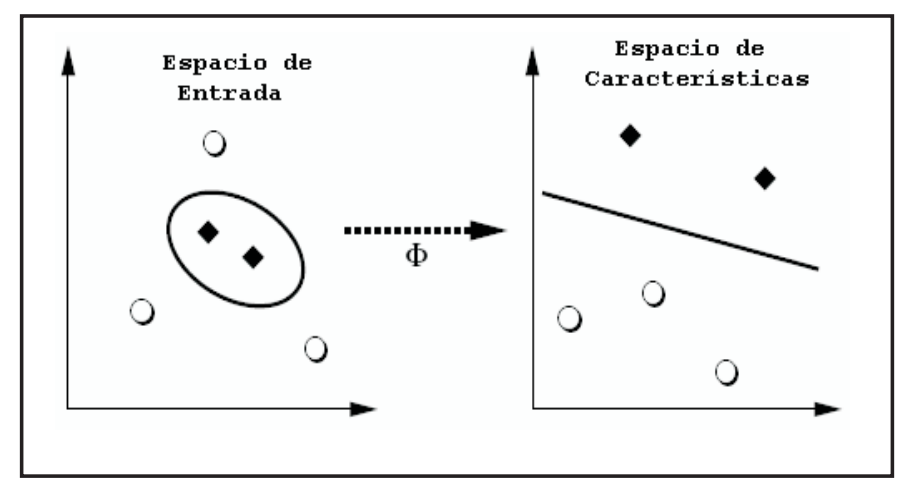

Figura 5. Transformación no lineal a priori del espacio de entrada a un espacio de características

Las máquinas de soporte vectorial son entrenadas a través del principio de inducción de minimización del riesgo estructural, con este principio se ha logrado implementar algoritmos de aprendizaje que logran controlar la habilidad de generalización de las máquinas de soporte vectorial, lo que ha permitido establecer un margen máximo de separación entre las clases (problemas de clasificación), además de hallar una superficie de separación entre las clases, que permite tener un intervalo de confianza óptimo para el grupo de funciones clasificadoras obtenidas en el proceso de entrenamiento; esto a diferencia del perceptrón multicapa que es entrenado a través del principio de minimización del riesgo empírico y donde se busca la generalización a través de lograr un mínimo error de entrenamiento [9] y [13].

Se debe contar con cierta cantidad de ejemplos para entrenamiento y otra cantidad de ejemplos para validar las máquinas de aprendizaje. Los 5900 ejemplos fueron divididos en cinco subconjuntos, cuatro de estos subconjuntos fueron utilizados para entrenar las máquinas de soporte vectorial con un kernel polinomial y un kernel de base radial, el subconjunto sobrante se utilizó como conjunto de validación, la Figura 6 ilustra un poco este método (holdon method). Este proceso se realizó cinco veces usando un subconjunto diferente de validación [14]. Los 210 Esquemas de extracción de características fueron sometidos a este proceso con el fin de determinar el grupo adecuado de ejemplos para entrenamiento y para validación.

Se seleccionaron los primeros 900 ejemplos de cada clase para entrenamiento y los 280 ejemplos finales para validación. Con el fin de comparar los resultados de clasificación y generalización de las máquinas de soporte vectorial, se seleccionó el perceptrón multicapa, de gran divulgación y de muy buenos resultados en diversas aplicaciones [15], [16] y [17]. Adicionalmente se compararon los resultados con un clasificador estadístico de tipo bayesiano.

El perceptrón multicapa tenía dos capas ocultas cuyos pesos eran de 50 cada una, función de transferencia de cada capa correspondiente a tansig, dos mil épocas y el algoritmo de retro propagación de error trainscg. Para la máquina de soporte vectorial los parámetros que mejores resultados arrojaron corresponden a una configuración con la función núcleo gausiana RBF, Gamma=1, C=50 y Coeff $=1$. Y para el clasificador bayesiano se emplea la función de distribución gausiana y los respectivos cinco índices que etiquetan cada clase.

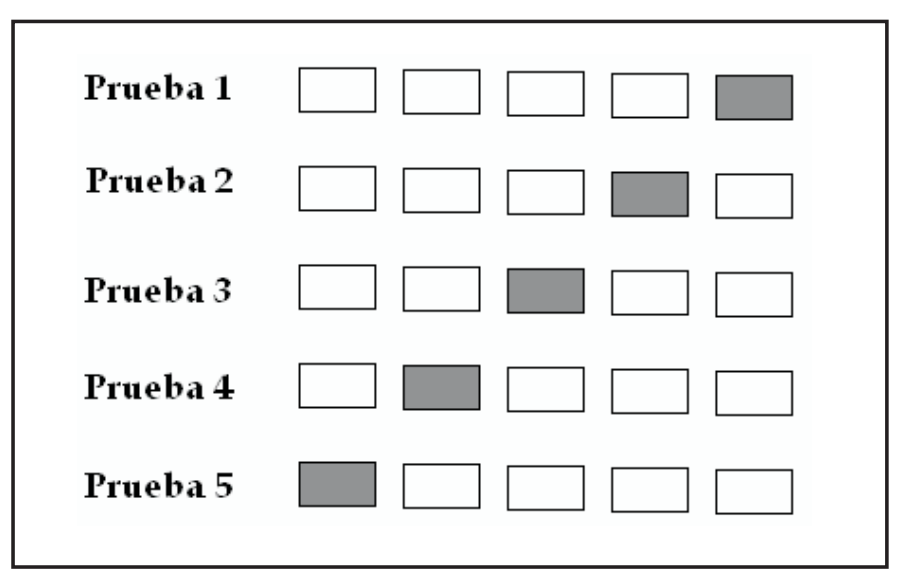

Figura 6. Método de validación cruzada dejar uno afuera (hold-out).

Sombreado aparece el subconjunto usado para validación

\section{RESULTADOS Y DISCUSIÓN}

La Tabla 4 muestra los mejores resultados obtenidos para la clasificación de cinco tipos de latidos, estos porcentajes de error se obtuvieron para el conjunto de validación. Cada uno de los Esquemas es explicado enseguida: 
Esquema 1: Aproximaciones, Niveles del 2 al 4, Todos los coeficientes de cada nivel, Wavelet Madre: Daubechies 4 (db4). Con 129 características.

Esquema 2: Aproximaciones, Nivel 1, Todos los coeficientes del nivel, Wavelet Madre: Daubechies 2 (db2). Con 129 características.

Esquema 3: Aproximaciones, Niveles del 2 al 4, Todos los coeficientes de cada nivel, Wavelet Madre: Symlet 5 (Sym5). Con 133 características.

Esquema 4: Aproximaciones, Nivel 1, Todos los coeficientes del nivel, Wavelet Madre: Daubechies 3 (db3). Con 130 características.

Esquema 5: Detalles, Niveles del 2 al 4, Todos los coeficientes de cada nivel, Wavelet Madre: Symlet 5 (Sym5). Con 133 características.

Los Esquemas 6, 7, 8, 9 y 10 corresponden a los mismos Esquemas mencionados anteriormente, pero tras la aplicación de PCA, seleccionando las primeras 15 componentes principales, es decir, al conjunto del Esquema 1 se le aplica PCA y se obtiene el conjunto del Esquema 6; al conjunto del Esquema 2 se le aplica PCA y se obtiene el conjunto del Esquema 7 y así sucesivamente...

Analizando los resultados de clasificación para los esquemas del 1 al 5 (sin PCA), se puede ver que el Esquema 1 presenta el menor error de validación tanto para la máquina de soporte vectorial (2.93\%) como para el perceptrón multicapa (3.63\%), lo que determina que los coeficientes de aproximación contienen información relevante y diferenciadora de cada conjunto de ejemplos a clasificar. Son resultados alentadores si se comparan con los presentados en otros artículos donde se mencionan errores de validación que oscilan entre un $2.4 \%$ y $6.67 \%$ [4] y [5], pero clasificando solo dos tipos de latidos cardiacos, mientras que en este trabajo se clasificaron cinco tipos. En general los resultados logrados tanto con las máquinas de soporte vectorial como con el perceptrón multicapa, indican que la metodología desarrollada cumple satisfactoriamente con las expectativas.

En el Esquema 5 se logra el mejor resultado con el clasificador bayesiano (11.72\%), sin embargo es poco alentador ya que están muy por debajo de los otros dos clasificadores; además el error de validación logrado en el Esquema 5 es aislado, ya que los demás están alrededor $78 \%$.
Tabla 4. Porcentaje de error para cada máquina de aprendizaje

\begin{tabular}{|c|c|c|c|}
\hline $\begin{array}{c}\text { Vector de } \\
\text { características }\end{array}$ & $\begin{array}{c}\text { Error (\%) } \\
\text { MSV }\end{array}$ & $\begin{array}{c}\text { Error (\%) } \\
\text { MLP }\end{array}$ & $\begin{array}{c}\text { Error (\%) } \\
\text { CB }\end{array}$ \\
\hline Esquema 1 & $\mathbf{2 . 9 3}$ & $\mathbf{3 . 6 3}$ & 79.85 \\
\hline Esquema 2 & 5.65 & 4.5 & 78.72 \\
\hline Esquema 3 & 4.43 & 4.29 & 79.74 \\
\hline Esquema 4 & 5.79 & 4.79 & 78.65 \\
\hline Esquema 5 & 3.22 & 4 & $\mathbf{1 1 . 7 2}$ \\
\hline Esquema 6 & $\mathbf{2 . 4 3}$ & 4.12 & $\mathbf{1 1 . 4 5}$ \\
\hline Esquema 7 & $\mathbf{2}$ & $\mathbf{3 . 8 6}$ & 14.74 \\
\hline Esquema 8 & $\mathbf{2 . 0 8}$ & 4.5 & 13.05 \\
\hline Esquema 9 & $\mathbf{1 . 9 3}$ & 4.08 & 15.72 \\
\hline Esquema 10 & 2.79 & 7.5 & 19.72 \\
\hline
\end{tabular}

MSV: Máquina de soporte vectorial ; MLP: Perceptrón multicapa; CB: Clasificador Bayesiano

Después de aplicar PCA a estos Esquemas se debe resaltar que los resultados mejoraron notablemente para el clasificador bayesiano, logrando un porcentaje de error mínimo de $11.45 \%$ para el Esquema 6 y un error promedio de validación de 14.93\%. Curiosamente, el Esquema 10, resultado de aplicar PCA al Esquema 5 (11.72\%), presentó un porcentaje de error mayor al del Esquema 5 (19.72\%), esto podría indicar que se pierde información relevante contenida en el conjunto de entrenamiento del esquema 5 al aplicarle PCA.

Igualmente, al aplicar PCA a los esquemas evaluados con las máquinas de soporte vectorial, se logró reducir los porcentajes de error para todos ellos, logrando el mejor caso con el vector de características descrito por el Esquema 9, el cual corresponde al nivel 1 de aproximaciones con Daubechies 3 (db3) (1.93\%). Se debe resaltar que éste efecto no se logró para el perceptrón multicapa, ya que en algunos casos se redujo el error (Esquema 2 y 4 ) pero en otros se incrementó (Esquema 1, 3 y 5).

Como se puede observar en la mayoría de resultados mostrados, la aplicación PCA constituye una mejora representativa en los resultados, logrando clasificaciones más confiables, lo que facilita el reconocimiento de este tipo de anormalidades cardiacas tras la eliminación de redundancia y de dimensionalidad en la información.

El perceptrón multicapa es una alternativa válida cuando se requiere la utilización de sistemas de aprendizaje, aunque según los resultados de éste estudio tienen una menor eficiencia que las máquinas de soporte vectorial, es probable que esta generalización tenga sus excepciones; debido a que la diferencia entre los resultados de dos clasificadores dista de $2 \%$ al $4 \%$. Con el clasificador bayesiano el cual es de 
aprendizaje debido al análisis previo de momentos estadísticos que realiza con los conjuntos proporcionados, pero en la Tabla 4 se observa que los porcentajes de error de validación son muy inferiores a los obtenidos por las máquinas de soporte vectorial y el perceptrón multicapa. Se esperaba un comportamiento mejor para algunos esquemas de extracción de características debido a la cantidad que se propusieron, sin embargo ningún resultado satisfizo las expectativas. Según la teoría que respalda este tipo de clasificadores se debe contar con una mayor cantidad de ejemplos de cada clase teniendo en cuenta la variabilidad de cada señal y distribuir la información entregada con una mejor metodología.

Si se comparan los resultados logrados con los publicados en [4], [5] y [18] cuyos porcentajes de error están entre el $0 \%$ y el $2 \%$ y los cuales también emplean técnicas para reducción de redundancia en la información, pero clasificando 2 , y en otros, 3 tipos de latidos cardiacos. Se observa que el trabajo desarrollado presenta resultados satisfactorios y que están dentro del margen de error registrado en otras publicaciones que emplean diferentes metodologías con el objetivo de clasificar latidos cardiacos. Uno de los pocos artículos donde se clasifican más de 5 tipos de latidos cardiacos es el [19] con 10 diferentes arritmias y latidos normales presentando un error mínimo de $4,56 \%$.

\section{CONCLUSIONES}

Las máquinas de soporte vectorial con función núcleo de base radial, son una excelente alternativa para la identificación de arritmias cardíacas. Mostraron una alta capacidad de generalización para esta tarea usando la transformada wavelet como metodología para la extracción de características y presentaron una gran compatibilidad con la técnica de análisis por componentes principales, seleccionando efectivamente las características e incrementando la eficiencia de la máquina de aprendizaje. No hay una diferencia sustancial entre los resultados obtenidos para las máquinas de soporte vectorial y los obtenidos para el perceptrón multicapa (está entre un 2 y $4 \%)$, lo que indica que posiblemente el perceptrón multicapa mejoré utilizando otras metodologías para extraer características, lo que sí es evidente, es la compatibilidad entre las máquinas de soporte vectorial y la técnica de análisis por componentes principales, ya que al aplicar esta técnica a los vectores de características, se logró siempre un error de clasificación menor para estas máquinas.

Con la selección efectiva de características, también se pudo observar como el clasificador bayesiano mejorabasustancialmente su capacidad de clasificación, hubo una mejoría representativa para los esquemas $6,7,8$ y 9 con respecto a los mostrados en los esquemas 1 al 4; pero el Esquema 10 obtuvo un porcentaje de error mayor (19.71\%) que el porcentaje de error para el Esquema 5 sin PCA (11.75\%), esto indica que la información contenida dentro de este vector de características es poco redundante y al aplicarle PCA se perdió información discriminante importante.

La transformada wavelet es un herramienta adecuada para el análisis de señales electrocardiográficas, permitiendo conocer características representativas de las señales tratadas propiciando una buena diferenciabilidad entre señales de diferentes tipos.

Aunque los resultados obtenidos fueron satisfactorios, vale la pena estudiar y analizar otras técnicas empleadas para el análisis y clasificación de latidos cardiacos, como es por ejemplo el establecimiento de vectores de características basados en la utilización de parámetros energéticos, temporales e inclusive proporcionando los eventos cardiacos sin una previa extracción de características; también se propone la exploración de otras técnicas de transformación para la extracción de características, como son los paquetes wavelet y la transformada wavelet compleja, que en la actualidad toman fuerza para aplicaciones en el tratamiento de señales bio-eléctricas.

\section{BIBLIOGRAFÍA}

(1) García GA, Velandia RA, Barón EM. Algoritmo de reducción de ruido en señales de electroencefalografía utilizando la DWT. Umbral científico [serie online] 2006 junio número 008; pag 34-40. Disponible en: http://redalyc.uaemex.mx/redalyc/pdf/304/30400805.pdf

(2) Tovar D, Orozco A, Muñoz P. Máquinas de Aprendizaje para la identificación de Arritmias Cardiacas. Memorias XIV simposio de tratamiento de señales, imágenes y visión artificial (XIV STSIVA); 2009 sept. 9-11; Pereira, Colombia.

(3) Cuesta F. Estudio de métodos para procesamiento y agrupación de señales electrocardiografícas. [Tesis doctoral]. Valencia (Esp): Universidad Politécnica de Valencia; 2001. 
(4) Rengifo C, Castellanos G, Henao R. Aprendizaje activo en la identificación de cardiopatía isquémica. grupo control y procesamiento de digital de señales. Memorias IX simposio de tratamiento de señales, imágenes y visión artificial (IX STSIVA); 2004 sept.; Manizales, Colombia.

(5) Montes V, Guarín C, Castellanos G. Extracción de características de ECG basadas en transformaciones no lineales y wavelets. Ingeniería e investigación 2005; Vol. 25; Numero 003: 39-48.

(6) Rivera J. Selección efectiva de características ECG mediante técnicas no lineales para identificación de patología IAM. [Tesis de Maestría]. Pereira (Col.): Universidad Tecnológica de Pereira; 2006.

(7) Cuesta F, Novak D, Perez JC, et al. Reducción del ruido en señales electrocardiográficas mediante la transformada Wavelet. Libro de Actas XVIII Congreso Anual de la Sociedad de Ingeniería Biomédica, CASEIB 2000. Cartagena, Colombia: 103-106.

(8) Sepulveda A, Castellanos G. Estimación de la frecuencia fundamental de señales de voz usando transformada wavelet. Scientia et technica 2004; Numero 24: 7-11

(9) Cardona JE, Ibargúen FJ, Muñoz PA. Máquinas de soporte vectorial para clasificación. 1 ed. Armenia (Q): Arte Imagen; 2007.

(10) Huff J. ECG Workout. Exercises in arrhythmia interpretation. 5 ed. Nueva York: Lippincott Williams \& Wilkins; 2006.

(11) Donoho D. De-noising by soft-thresholding. IEEE Transactions on Information Theory 1995; Vol 41 issue 3: 613-627.

(12) Yunfeng W, Rangaraj M, Rangayyan B, Yachao Z, Sin-chun N. Filtering electrocardiographic signals using an unbiased and normalized adaptive noise reduction system. Medical Engineering \& Physics 2009; No. 31: 17-26.

(13) Vapnik V. The nature of statisctical learning theory. 2 ed. Nueva York: Springer verlag. 2.000

(14) Haykin S. Neural networks. A comprehensive foundation. New York: Prentice Hall. 1996

(15) Muñoz PA, Ibarguen FJ, Cardona JE. Sistema para el reconocimiento fuera de línea de caracteres manuscritos. Revista de investigaciones Universidad del Quindío 2007; Numero 17: 189-203.

(16) Balomenos T, Kapantkicos K, Tsapasoulis N. On the use of ECG waveforms for health status estimation. 2002. Disponible: http://www.image.ece.ntua.gr/papers/231.pdf

(17) De Chazal P, McDarby G, Reilly R. A wavelet based classifier of the electrocardiogram. Proceeding of the european medical biology conference 1999. Viena, Austria.

(18) Giraldo E, Fetecua J, Rodríguez A. Caracterización adaptativa señales ECG a partir de descomposición por wavelet packet. Scientia et technica 2006; Numero 32: 19-23

(19) Orozco M, Castellanos G, Orozco R. Extracción de características usando wavelets en la identificación de patologías de ECG. Memorias I Congreso de Bioingeniería e Ingeniería Biomédica 2003. Medellín, Colombia. 